\title{
Solutions of nonlinear fractional coupled Hirota-Satsuma-KdV Equation
}

\author{
Runqing Cui \\ School of Mathematics and Informatics, Henan Polytechnic University, Jiaozuo 454003, China \\ Igdcuirunqing@yeah.net
}

\section{ABSTRACT:}

Our interest in the present work is in implementing the FPSM to stress it power in handing the nonlinear fractional coupled Hirota-Satsuma-KdV Equation. The approximate analytical solution of this type equations are obtained.

\section{Keywords:}

nonlinear fractional coupled Hirota-Satsuma-KdV Equation; fractional power series method; Caputo fractional derivative

\section{INTRODUCTION}

During this last decades the nonlinear fractional differential equation have been applied in various scientific and engineering fields, such as electromagnetic theory, fluid mechanics, biology, solid state physics, chemical physics and geochemistry etc[1-16]. In most cases, it is very difficult to obtain exact solutions. So these types of equations must be solved by any numerical methods or approximate methods. Many approaches for the solution of fractional differential equations have been proposed. These methods include the direct algebraic method [4-5], Jacobi elliptic function method [6], tanh-function method [7], variational iteration method and homotopy perturbation method [8,9], Adomian decomposition method [11], sine-cosine method [12], homotopy analysis method [13], the differential transform method [14], and fractional power series method(FPSM)[15].

The FPSM is a powerful tool for solving linear and nonlinear problems. Our interest in the present work is in implementing the FPSM to stress it power in handing the following nonlinear fractional coupled Hirota-Satsuma-KdV Equation:

$$
\begin{aligned}
& D_{t}^{\alpha} u=\frac{1}{4} \frac{\partial^{3} u}{\partial x^{3}}+3 u \frac{\partial u}{\partial x}-6 v \frac{\partial v}{\partial x}, 0<\alpha \leq 1, \\
& D_{t}^{\alpha} v=-\frac{1}{2} \frac{\partial^{3} v}{\partial x^{3}}-3 u \frac{\partial u}{\partial x}
\end{aligned}
$$

subject to the conditions

$$
u(x, 0)=-\tanh ^{2} x, v(x, 0)=\tanh x
$$

where $D^{\alpha}(1 \geq \alpha>0)$ denote the Caputo fractional derivative of order $\alpha$.

The Eqs.(1) and (2) arise in many scientific applications such as quantum mechanics and plasma physics [1-3]. It is well known that wave phenomena of plasma are modeled by kind tanh solution.

The present paper has been organized as follows. In section 2, we introduce the basic definitions and properties of fractional calculus and the FPSM is described. In section 3 the FPSM is applied for the EqS.(1) and (2). Conclusion is 
presented in Section 4.

\section{BASIC DEFINITIONS}

In this section, we will introduce notations, definitions and some useful lemmas, which play an important role in obtaining the main results of this paper. We begin with some basic definitions [15].

Definition 1. A real function $f(x), x>0$ is said to be in the space $C_{\mu}, \mu \in R$ if there exists a real number $p>\mu$, such that $f(x)=x^{p} f_{1}(x)$ where $f_{1}(x) \in C[0, \infty)$ and it is said to be in the space $C_{n}$ if and only if $f^{(n)} \in C_{\mu}, n \in N$.

The Riemann-Liouville fractional integral operator is defined as follows:

Definition 2. The Riemann-Liouville fractional integral operator of order $\alpha>0$ of a function $f(x) \in C_{\mu}, \mu \geq-1$ is defined as:

$$
\begin{aligned}
& J^{\alpha} f(x)=\frac{1}{\Gamma(\alpha)} \int_{0}^{x}(x-s)^{\alpha-1} f(s) d s, \\
& J^{0} f(x)=f(x) .
\end{aligned}
$$

Properties of the operator $J^{\alpha}$ can be found in and we mention only the following: For $\alpha, \beta \geq 0, x>0$ and $\gamma>-1$ :

$$
\begin{gathered}
J^{\alpha} J^{\beta} f(x)=J^{\alpha+\beta} f(x), \\
J^{\alpha} J^{\beta} f(x)=J^{\beta} J^{\alpha} f(x), \\
J^{\alpha}\left(x^{\gamma}\right)=\frac{\Gamma(\gamma+1)}{\Gamma(1+\alpha+\gamma)} x^{\gamma+\alpha} .
\end{gathered}
$$

Definition 3. The fractional derivative of $f(x)$ in Caputo sense is defined as:

$$
D^{\alpha} f(x)=\frac{1}{\Gamma(m-\alpha)} \int_{0}^{x}(x-s)^{m-\alpha-1} f^{(m)}(s) d s
$$

for $m-1<\alpha \leq m, m \in N^{+}, x>0$ and $f \in C_{-1}^{m}$.

We recall here two of its basic properties:

$$
D^{\alpha} J^{\alpha} f(x)=f(x),
$$




$$
J^{\alpha} D^{\alpha} f(x)=f(x)-\sum_{k=0}^{m-1} f^{(k)}\left(0^{+}\right) \frac{x^{k}}{k !}, x>0 .
$$

Definition 4. A power series representation of the form

$$
\sum_{n=0}^{\infty} c_{n}\left(t-t_{0}\right)^{n \alpha}=c_{0}+c_{1}\left(t-t_{0}\right)^{\alpha}+c_{2}\left(t-t_{0}\right)^{2 \alpha}+\cdots
$$

where $0 \leq m-1<\alpha \leq m, m \in N^{+}$and $t \geq t_{0}$ is called a fractional power series (FPS) about $t_{0}$, where $t$ is a variable and $c_{n}$ are the coeffients of the series.

We also need the following property:

Lemma 1. Suppose that the FPS $\sum_{n=0}^{\infty} c_{n} t^{n \alpha}$ has radius of convergence $R>0$. If $f(t)$ is a function defined by $f(t)=\sum_{n=0}^{\infty} c_{n} t^{n \alpha}$ on $0 \leq t<R$, then for $m-1<\alpha \leq m$ and $0 \leq t<R$, we have:

$$
D^{\alpha} f(t)=\sum_{n=0}^{\infty} c_{n} \frac{\Gamma(n \alpha+1)}{\Gamma((n-1) \alpha+1)} t^{(n-1) \alpha}
$$

\section{THE SOLUTIONS OF Eqs. (1) AND (2)}

To solve the Eqs. (1) and (2) by FPSM, suppose that the solution of (1) and (2) takes the form:

$$
\begin{aligned}
& u(x, t)=\sum_{k=0}^{\infty} a_{k}(x) t^{\alpha k}=a_{0}(x)+a_{1}(x) t^{\alpha}+a_{2}(x) t^{2 \alpha}+\cdots \\
& v(x, t)=\sum_{k=0}^{\infty} b_{k}(x) t^{\alpha k}=b_{0}(x)+b_{1}(x) t^{\alpha}+b_{2}(x) t^{2 \alpha}+\cdots
\end{aligned}
$$

Using (3), we have

$$
a_{0}(x)=-\tanh ^{2} x, b_{0}(x)=\tanh x .
$$

Next we determine the $a_{k}(x), b_{k}(x)(k=1,2, \cdots)$.

From Lemma 1, we obtain

$$
D_{x}^{\alpha} u(x, t)=\sum_{k=1}^{\infty} \frac{a_{k}(x) \Gamma(\alpha k+1)}{\Gamma(\alpha(k-1)+1)} t^{\alpha(k-1)} .
$$




$$
D_{x}^{\alpha} v(x, t)=\sum_{k=1}^{\infty} \frac{b_{k}(x) \Gamma(\alpha k+1)}{\Gamma(\alpha(k-1)+1)} t^{\alpha(k-1)} .
$$

Substituting (6) and (7) into (4), and comparing the coefficients of $t^{\alpha}$ in the both side, we get

$$
\begin{gathered}
a_{1}(x)=\frac{8 \Gamma(\alpha) e^{2 x}\left(1-e^{2 x}\right)}{\Gamma(\alpha+1)\left(1+e^{2 x}\right)^{3}}, \\
b_{1}(x)=\frac{4 \Gamma(\alpha) e^{2 x}}{\Gamma(\alpha+1)\left(1+e^{2 x}\right)^{2}}, \\
a_{2}(x)=\frac{16 e^{2 x} \Gamma(\alpha+1)\left(1-4 e^{2 x}+e^{4 x}\right)}{\Gamma(2 \alpha+1)\left(1+e^{2 x}\right)^{4}}, \\
a_{3}(x)=\frac{16 e^{2 x} \Gamma(2 \alpha+1)\left(1-e^{2 x}\right)\left(1-10 e^{2 x}+e^{4 x}\right)}{\Gamma(3 \alpha+1)\left(1+e^{2 x}\right)^{5}}, \\
b_{2}(x)=\frac{8 e^{2 x} \Gamma(\alpha+1)\left(1-2 e^{2 x}\right)}{\Gamma(2 \alpha+1)\left(1+e^{2 x}\right)^{3}}, \\
a_{4}(x)=\frac{32 e^{2 x} \Gamma(3 \alpha+1)\left(1-26 e^{2 x}+66 e^{4 x}-26 e^{6 x}+e^{8 x}\right)}{3 \Gamma(4 \alpha+1)\left(1+e^{2 x}\right)^{6}}, \\
b_{3}(x)=\frac{8 e^{2 x} \Gamma(2 \alpha+1)\left(1-4 e^{2 x}+e^{4 x}\right)}{\Gamma(3 \alpha+1)\left(1+e^{2 x}\right)^{4}}, \\
\frac{16(4 \alpha+1)\left(1+e^{2 x}\right)^{5}}{\Gamma(3 \alpha+1)\left(1-11 e^{2 x}+11 e^{4 x}-e^{6 x}\right)},
\end{gathered}
$$

and so on.

Thus we obtain the solution

$$
\begin{gathered}
u(x, t)=-\tanh ^{2} x+\frac{8 \Gamma(\alpha) e^{2 x}\left(1-e^{2 x}\right)}{\Gamma(\alpha+1)\left(1+e^{2 x}\right)^{3}} t^{\alpha} \\
+\frac{16 e^{2 x} \Gamma(\alpha+1)\left(1-4 e^{2 x}+e^{4 x}\right)}{\Gamma(2 \alpha+1)\left(1+e^{2 x}\right)^{4}} t^{2 \alpha}
\end{gathered}
$$




$$
\begin{aligned}
& +\frac{16 e^{2 x} \Gamma(2 \alpha+1)\left(1-e^{2 x}\right)\left(1-10 e^{2 x}+e^{4 x}\right)}{\Gamma(3 \alpha+1)\left(1+e^{2 x}\right)^{5}} t^{3 \alpha} \\
& +\frac{32 e^{2 x} \Gamma(3 \alpha+1)\left(1-26 e^{2 x}+66 e^{4 x}-26 e^{6 x}+e^{8 x}\right)}{3 \Gamma(4 \alpha+1)\left(1+e^{2 x}\right)^{6}} t^{4 \alpha}+\cdots \\
& v(x, t)=\tanh x+\frac{4 \Gamma(\alpha) e^{2 x}}{\Gamma(\alpha+1)\left(1+e^{2 x}\right)^{2}} t^{\alpha} \\
& +\frac{8 e^{2 x} \Gamma(\alpha+1)\left(1-2 e^{2 x}\right)}{\Gamma(2 \alpha+1)\left(1+e^{2 x}\right)^{3}} t^{2 \alpha} \\
& +\frac{8 e^{2 x} \Gamma(2 \alpha+1)\left(1-4 e^{2 x}+e^{4 x}\right)}{\Gamma(3 \alpha+1)\left(1+e^{2 x}\right)^{4}} t^{3 \alpha} \\
& +\frac{16 e^{2 x} \Gamma(3 \alpha+1)\left(1-11 e^{2 x}+11 e^{4 x}-e^{6 x}\right)}{3 \Gamma(4 \alpha+1)\left(1+e^{2 x}\right)^{5}} t^{4 \alpha}+\cdots
\end{aligned}
$$

The exact solution to the problem (1)-(3) when $\alpha=1$ are given as:

$$
\begin{aligned}
& u(x, t)=-\tanh ^{2}(x+t), \\
& v(x, t)=\tanh (x+t) .
\end{aligned}
$$

\section{CONCLUSION}

The FPSM is a powerful tool for solving the nonlinear fractional coupled Hirota-Satsuma-KdV Equation. Our results show that the FPSM is simple, direct and effective.

\section{REFERENCES}

[1] Ganji, D. D., and M. Rafei. Solitary wave solutions for a generalized Hirota-Satsuma coupled KdV equation by homotopy perturbation method. Physics Letters A 356.2(2006):131-137.

[2] Abazari, Reza, and M. Abazari. Numerical simulation of generalized Hirota-Satsuma coupled KdV equation by RDTM and comparison with DTM.Communications in Nonlinear Science \& Numerical Simulation17.2(2012):619-629.

[3] Liu, Jincun, and H. Li. Approximate Analytic Solutions of Time-Fractional Hirota-Satsuma Coupled KdV Equation and Coupled MKdV Equation. Abstract and Applied Analysis,2013,(2013-3-24)2013.2013(2013):103-128.

[4] Seadawy, A. R. The Solutions of the Boussinesq and Generalized Fifth-Order KdV Equations by Using the Direct Algebraic Method. Applied Mathematical Sciences 6.82(2012):4081-4090.

[5] Yan, Wang Ji, and W. X. Ren. A direct algebraic method to calculate the sensitivity of element modal strain energy. International Journal for Numerical Methods in Biomedical Engineering 27.5(2011):694-710.

[6] Parkes, E. J., B. R. Duffy, and P. C. Abbott. The Jacobi elliptic-function method for finding periodic-wave solutions to 
nonlinear evolution equations. Physics Letters A 295.5-6(2002):280-286.

[7] Ali, A. H. A. The modified extended tanh-function method for solving coupled MKdV and coupled Hirota-Satsuma coupled KdV equations. Physics Letters A 363.5-6(2007):420-425.

[8] He, Ji Huan. Variational iteration method - a kind of non-linear analytical technique: some examples. International Journal of Non-Linear Mechanics 34.4(1999):699-708.

[9] Chowdhury, Md., A comparison between the modified homotopy perturbation method and adomian decomposition method for solving nonlinear heat transfer equations, Journal of Applied Sciences, 11 (2011):1416-1420.

[10] He, Ji Huan. Homotopy perturbation method: a new nonlinear analytical technique ,Applied Mathematics \& Computation 135.1(2003):73-79.

[11] Ray, Santanu Saha. A new approach for the application of Adomian decomposition method for the solution of fractional space diffusion equation with insulated ends. Applied Mathematics \& Computation202.2(2008):544-549.

[12] Wazwaz, Abdul Majid. "The sine-cosine method for obtaining solutions with compact and noncompact structures. Applied Mathematics \& Computation 159.2(2004):559-576.

[13] Liao, Shijun, Homotopy analysis method: a new analytical technique for nonlinear problems, Communications in Nonlinear Science and Numerical Simulation, 2 (1997),2, pp.95-100.

[14] Arikoglu, Aytac, and I. Ozkol. Solution of fractional differential equations by using differential transform method. Chaos Solitons \& Fractals34.5(2007):1473-1481.

[15] Kurulay, Muhammet, and M. Bayram. Power series method for linear partial differential equations of fractional order. Commun.math.appl1.2(2013):71-76.

[16] Hilfer, Rudolf, ed., Applications of fractional calculus in physics, World Scientific, 2000.

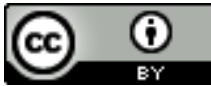

This work is licensed under a Creative Commons Attribution 4.0 International License. 